\title{
Medical products - who is responsible?
}

\section{Editorial}

There are many Medical Products on the market in order to improve the state of patients. The wide majority of these have no problems. By way of contrast the admission of a medical drug, it is very easy to start a medical product on the market. In short, the approval of a Medical Authority and the CE mark of the producer are sufficient. There will be no consulting of a material specialist at all and definitely no vigilance by a Medical Authority, according to their self-image. The question will be, whether the community can afford this idling of the Medical Authorities in the future ... Several examples should throw lights on to this severe problem. Among others, two details according the treatment of dialysis are worthy to make transparent. At first, this is the complex of mechanical haemolysis today. Indeed, it appears seldom. These poor patients may change suddenly into a state of critical illness, when a considerable raise of resistance will appear in the first part of the extracorporeal circuit of a dialysis.

The problem is, that there may be no pressure monitoring in this part of the extracorporeal circuit (no BPE (Blood Pressure Entry)) in order to stop a critical treatment. These conditions of haemolysis may take place, by not-registration of a kinking of the bloodline (during the preparation of the dialysis) or by a not realized totally thrombosis of the dialyzer. These patients will transfer to the ICU because of their general condition. The diagnosis of haemolysis often will do, when the blood samples will investigate. According to the schedule of dialysis, the used extracorporeal bloodline had wasted often before the diagnosis of haemolysis had done. In 2012, haemolysis happened in 25 patients in Germany with an ordinary correct standard bloodline. All patients had transferred and treated in the ICU. The problem had discussed by the medical society and by the producer. Unfortunately - you will not believe this - this problem had not well understood. According to the fashion e.g. of skirts, trousers or curtains, the length of the bloodline had changed - whenever this was not the problem, as the used standard bloodline had correct measures. The BPI measurement not had introduced obligatory.

This measurement however does prevent haemolysis, when there is a critical mechanical resistance either in the dialyzer or in the bloodline in front of the dialyzer. It is not a question of the costs and even not of the monitoring (> TMP is no longer necessary, due to UF-coefficients of more than $60 \mathrm{ml} / \mathrm{mm} \mathrm{Hg} / \mathrm{min}$. in high-flux dialysis, as today there does exist a second way of the monitoring of the ultrafiltration volume). It is a problem to understand the conditions of mechanical haemolysis today. This is the remaining question concerning the vigilance of a medical product by the Medical Authorities. Idling is the word concerning the vigilance of a medical product ... The other big theme of critical medical products is dialysis fluids. In 1993, there was a test infusion of bicarbonate for bag hemofiltration without any acidification. My calculations I had sent to the former Medical Authority, the BGA in Berlin (Germany). This BGA had really acted in alert condition: They confirmed my calculations. The required acidification had added ... - the chemist of
Volume 4 Issue 4 - 2017

\author{
Thomas Ryzlewicz \\ Department of Nephrology, Dialysis Centre, Germany
}

Correspondence: Thomas Ryzlewicz, Department of Nephrology, Dialysis Centre,Via Medis D-01589 Riesa, RobertKoch-Strasse 30, Germany, Tel 08055।8 91 28, Fax 08055189129, Email thomas.ryzlewicz@web.de

Received: April 14, 2017 | Published: April 19, 2017

the producer had done a mistake (by 2 ten-potencies) ... - later on the BGA had closed by the ministry of health (> problems to detect HIV antibodies in stored blood).

Continuing the problem of dialysis fluid. In 1978, bicarbonate dialysis fluid had introduced with the acidification of $3 \mathrm{mmol} / \mathrm{l}$ acetate in order to reduce the calcification of this prescription. As there does exist a continuous calcification, the monitor will descaled after each dialysis treatment. The patient has his own calcification problem by the ingestion of orally phosphate with the nutrition. There is a well-known big rate of morbidity and mortality of CKD-5 patients, matching for this the calcifications of coronary vessel and of heart valves. There is a big lamentation of this medical situation by the medical societies. The context of the calcification of dialysis fluid had not noticed, as the problem of the calcifying dialysis fluid (A-component with acidification of $3 \mathrm{mmol} / \mathrm{l}$ acetate) not had recognized. - Since several years, there does exist prescriptions with equimolar citrate acidification. As they have the same mval/l content concerning acids, there is the same amount of $\mathrm{CO}_{2}$ production as in the prescription with $3 \mathrm{mmol} / \mathrm{l}$ acetate. Citrate has a second way of working inside, the chelate binding.

By this, the problematic cat-ions (calcium and magnesium) will bind, so they cannot take part in the calcification. A-components with citrate acidification are admitted medical products for dialysis. The problem: Medical doctors, opinion leaders and Medical Authorities do never understand a complex problem of chemical solubility. Medical Authorities always had casted with medical doctors. There is the requirement to stop the dialysis concentrate for bicarbonate dialysis with $3 \mathrm{mmol} / \mathrm{l}$ acetate acidification because of the calcification in the prescription. We do not need Medical Authorities with idling concerning vigilance of medical products. They should consulted by material specialists (> e.g. chemists in this case). The way of idling concerning the vigilance, the community does not need. That is why, the Medical Authorities, responsible for medical products, should send into happily retirement - because of medical problems of the patients... 